\title{
Double Distal Perfusion Catheters for Severe Limb Ischemia on the IABP Side in Patients Who Received Femoro-Femoral VA-ECMO With IABP
}

\author{
Meng Xin, Liangshan Wang, Xiaqiu Tian, Dengbang Hou, Hong Wang, Jiangang Wang, \\ Ming Jia and Xiaotong Hou* \\ Center for Cardiac Intensive Care, Beijing Anzhen Hospital, Capital Medical University, Beijing, China
}

\section{OPEN ACCESS}

Edited by:

Samir G. Sakka,

Gemeinschaftsklinikum Mittelrhein

gGmbH, Germany

Reviewed by:

Ying $\mathrm{Su}$,

Fudan University, China

Xin Xu,

Zhejiang University, China

*Correspondence:

Xiaotong Hou

xt.hou@ccmu.edu.cn

Specialty section:

This article was submitted to

Intensive Care Medicine and

Anesthesiology,

a section of the journal

Frontiers in Medicine

Received: 08 April 2021 Accepted: 04 August 2021 Published: 25 August 2021

Citation:

Xin M, Wang L, Tian X, Hou D, Wang $H$, Wang J, Jia $M$ and Hou $X$ (2021) Double Distal Perfusion

Catheters for Severe Limb Ischemia on the IABP Side in Patients Who Received Femoro-Femoral VA-ECMO With IABP. Front. Med. 8:692399. doi: 10.3389/fmed.2021.692399
Background: Limited research is available on the pattern of double distal perfusion catheters in patients on venoarterial extracorporeal membrane oxygenation (VA-ECMO) with an intra-aortic balloon pump(IABP). Here, we compared the outcomes of a double distal perfusion catheter and conventional treatment in patients who received VA-ECMO with IABP and had severe lower limb ischemia on the IABP side.

Methods: We reviewed the data of 15 adult patients with postcardiotomy cardiogenic shock who received VA-ECMO via femoral cannulation combined with an IABP in the contralateral artery that was complicated with severe acute limb ischemia (ALI) on the same side as the IABP between January 2004 and December 2016. Patients underwent symptomatic treatment (conventional group, $n=9$ ) and double distal perfusion catheterization treatment (DDPC group, $n=6$ ). ALI was monitored using near-infrared spectroscopy placed on both calves after double distal perfusion catheters. The outcomes were compared.

Results: All 6 patients who underwent double distal perfusion catheters were successfully decannulated without the development of osteofascial compartment syndrome, amputation, or bleeding and infection of the double distal perfusion catheters. The number of patients who weaned from extracorporeal membrane oxygenation successfully in the DDPC and conventional groups was $6(100 \%)$ and $3(33 \%, p=0.028)$, respectively. The in-hospital mortality rates were $17 \%$ and $89 \%$ for the DDPC and conventional groups, respectively $(p=0.011)$.

Conclusions: DDPC can be considered a strategy for severe limb ischemia on the IABP side in patients who received femoro-femoral VA-ECMO with IABP.

Keywords: distal perfusion catheter, extracorporeal membrane oxygenation, intra-aortic balloon pump, osteofascial compartment syndrome, severe limb ischemia 


\section{INTRODUCTION}

Extracorporeal membrane oxygenation (ECMO) via femoral arteriovenous cannulation is an established option for adult patients with refractory cardiogenic shock after cardiac surgery. However, acute limb ischemia (ALI) is a common complication and sometimes requires fasciotomy or amputation and is a significant predictor of mortality $(1,2)$. Although it is controversial whether patients with intra-aortic balloon pumps (IABPs) combined with ECMO have better prognoses $(3,4)$, they might have an increased risk of ALI (5). ALI is a complication on both the ipsilateral side of the arterial cannula (ECMO side) as well as the contralateral side (IABP side). Conservative treatment is often ineffective in patients with severe ALI, and IABP may even need to be removed. Insertion of a distal catheter may be an effective means to improve the blood supply of the ischemic lower extremity and reserve IABP.

We describe 3 types of establishing perfusion catheters to relieve distal ischemia (Figure 1). The oxygenated blood is diverted from the arterial cannula to the distal limb of the ECMO side using a perfusion catheter, called the ECMO side distal perfusion catheter (EDPC). Some centers insert EDPC as a preventive routine to decrease limb ischemia $(2,6)$, while others implant it as treatment following ALI $(7,8)$. When patients with EDPC develop IABP side limb ischemia, another distal perfusion catheter is inserted distally on the IABP side, called a double distal perfusion catheter (DDPC). In the present study, we compared the outcomes of DDPC and conventional treatment in ECMO patients with ALI on the IABP side.

\section{METHODS}

\section{Study Population}

Between January 2004 and December 2016, 451 patients required VA-ECMO support following postcardiotomy cardiogenic shock (PCS), of which 245 patients required combined treatment with IABP. Fifteen patients $(6 \%)$ who were diagnosed with severe ALI on the IABP side were retrospectively enrolled in this study. Before January 2015, 9 patients underwent conventional treatment. Since January 2015, 6 patients underwent treatment with DDPC. Severe ALI was classified according to the Rutherford system as IIA, IIB or III (9). Category I was excluded because of better prognosis. The study was approved by the institutional ethics committee/review board of the Beijing Anzhen Hospital, Capital Medical University, and the requirement for informed patient consent was waived in view of the retrospective nature of the study.

\section{ECMO Implantation Techniques}

The VA-ECMO was placed by trained ECMO team members. ECMO cannulae (Biomedicus, Medtronic; Minneapolis, MN,

Abbreviations: ABI, Ankle-brachial index; DDPC, Double distal perfusion catheter; EDPC, Extracorporeal membrane oxygenation side distal perfusion catheter; IDPC, Intra-aortic balloon pump side perfusion catheter; IABP, Intraaortic balloon pumps; PCS, Postcardiotomy cardiogenic shock; SLI, Severe limb ischemia; ECMO, Extracorporeal membrane oxygenation; $\mathrm{StO}_{2}$, Tissue saturation; ICU, Intensive care unit; SOFA, Sequential organ failure assessment.
USA) were inserted through the femoral artery and femoral vein. A 6-Fr EDPC was inserted at the time of ECMO initiation to preserve limb perfusion. An IABP catheter (Datascope Corp., Fairfeld, NJ, USA) was placed percutaneously through the contralateral femoral artery.

\section{Patient Management}

Detailed management strategies for patients have been previously described (10). ECMO blood flow was adjusted to maintain a mixed venous oxygen saturation $(\mathrm{SvO} 2)$ level of $70 \%$. Blood circulation of the lower limbs was observed continuously by trained ICU staff during ECMO support. Medial or/and lateral incisions of a minimum of $15 \mathrm{~cm}$ were made when acute compartment syndrome developed (intracompartmental pressure ICP $>25 \mathrm{mmHg}$ ). Amputation was considered when ischemic tissue was subjected to unmanageable infections and when ischemic rest pain or tissue loss could not be restored by any surgical or non-surgical approaches.

A heparin bolus (5,000 IU) was injected before ECMO insertion. After surgical bleeding was controlled, unfractionated heparin was infused continuously as early as possible to maintain an activated clotting time of $160-180 \mathrm{~s}$. When SLI occurred on the IABP side, intravenous prostaglandin therapy or sympathicolysis was initiated. The vasoconstrictors were reduced gradually following hemodynamic stability. Upon failure to relieve ischemia, IABP was removed (conventional group). After January 2015, we began to insert DDPC in such patients (DDPC group).

\section{DDPC Insertion}

A $2-3-\mathrm{cm}$ incision was made $1 \mathrm{~cm}$ below the midpoint of the groin. The vasculo-neural sheath was dissected after the subcutaneous tissues and muscles were released. A 6-Fr distal perfusion catheter (Transradial Kit, Cordis Corporation, Miami Lakes, FL, USA) was inserted into the superficial femoral artery at the end of the IABP artery. The distal perfusion catheter was connected to the side hole of the arterial cannula of the ECMO circuit. DDPC decannulation was performed while ECMO was weaning.

\section{NIRS}

Continuous monitoring of limb perfusion began immediately after ALI was diagnosed and measured using bilateral nearinfrared spectroscopy (NIRS). The Oximeter sensor pads were placed on the bilateral lower limbs midway between the knee and ankle.

\section{Data Collection}

All clinical variables of patients were recorded in our institutional database. Tissue saturation ( $\mathrm{StO} 2)$ was detected using nearinfrared spectroscopy in the ICU, and persistent ALI was diagnosed until stable conditions were achieved. In addition to lactate and muscle injury markers, $\mathrm{StO} 2$ was recorded when ALI was diagnosed 6 and $24 \mathrm{~h}$ later. Patients also underwent measurement of the ankle brachial index (ABI) of both legs after DDPC was placed and during the follow-up measurements until ECMO and IABP were removed. Clinical indications of 


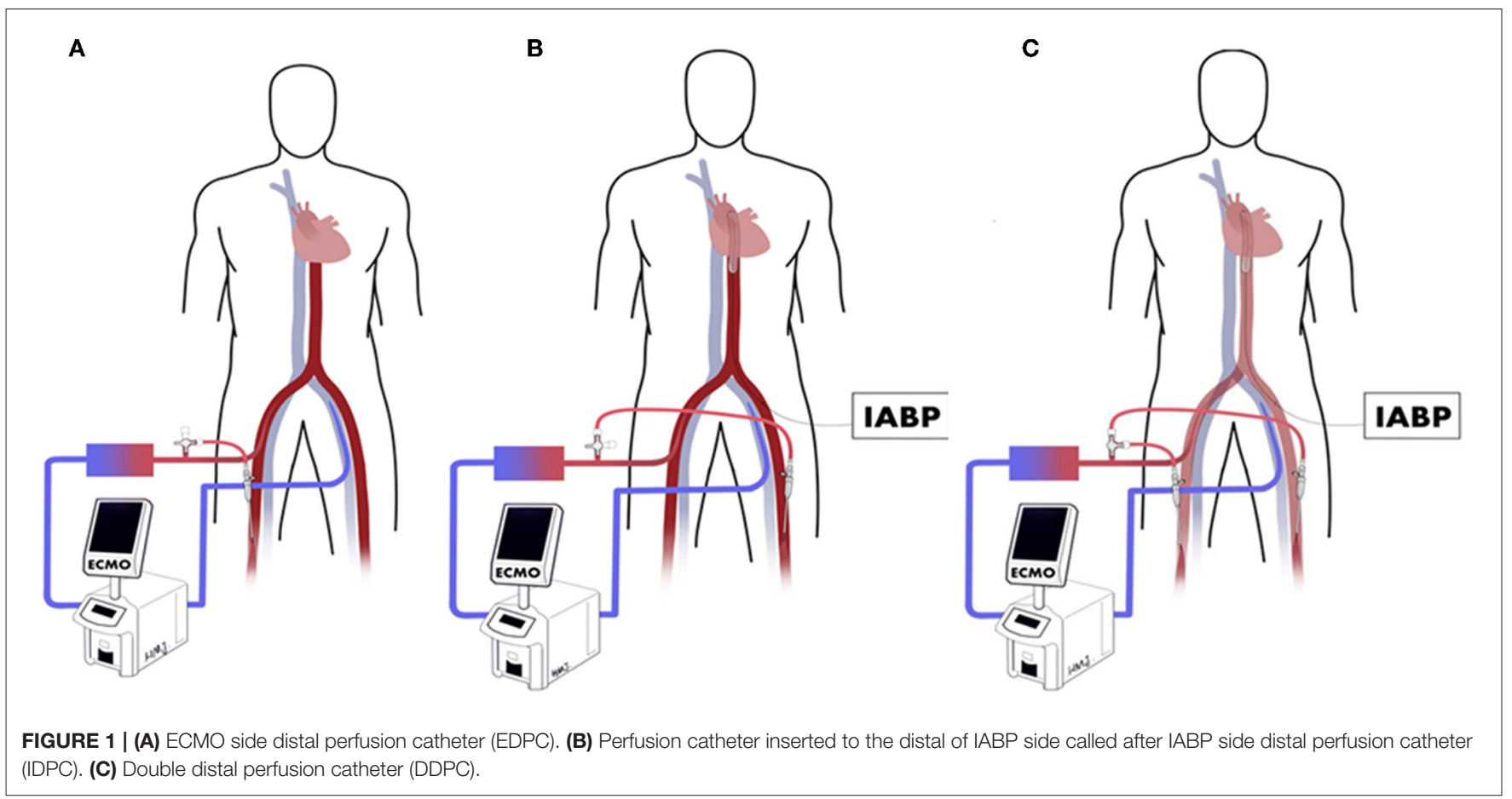

TABLE 1 | Demographics and clinical characteristics.

\begin{tabular}{lcc}
\hline \multirow{2}{*}{ Variables } & \multicolumn{2}{c}{ No. (\%) or median (interquartile range) } \\
\cline { 2 - 3 } & DDPC (6) & Conventional (9) \\
\hline Age, years & $48.2(16-68)$ & $54.1(36-63)$ \\
Male & $83.3 \%$ & $77.8 \%$ \\
BM! & $27.2(22.9-31.5)$ & $24.7(20.1-30.2)$ \\
Etiology & & $55.6 \%$ \\
CAD & $50 \%$ & $11.1 \%$ \\
VHD & $50 \%$ & $22.2 \%$ \\
AD & $0 \%$ & $11.1 \%$ \\
CHD & $0 \%$ & $33.3 \%$ \\
Complications & & $33.3 \%$ \\
Hypertension & $55.6 \%$ & $66.7 \%$ \\
Diabetes mellitus & $44.4 \%$ & $16.7 \%$ \\
Smoking history & $55.6 \%$ & $12.7(11-15)$ \\
Femoral stenosis history & $22.2 \%$ & $54.4(40-66)$ \\
SOFA scores (when ALI was diagnosed) & $12.9(12-14)$ & $3.6(3.5-3.9)$ \\
Inotrope scores (when ALI was diagnosed) & $61.7(43-78)$ & 0.32 \\
ECMO Flow (LPM) (when ALI was diagnosed) & $3.5(3.2-3.8)$ & 0.54
\end{tabular}

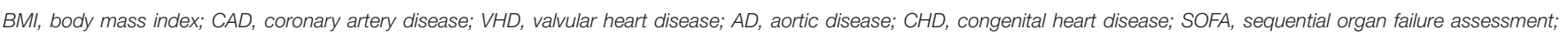

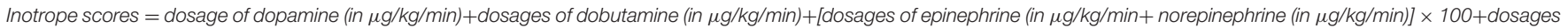
of pituitrin (in u/min) × 100+dosages of milrinone (in $\mu \mathrm{g} / \mathrm{kg} / \mathrm{min}) \times 15$.

hypoperfusion were also recorded, including cold limbs, mottled skin, and pulseless Doppler signaling after DDPC.

\section{Statistical Analysis}

SPSS software (IBM Corp., SPSS Version 25, Armonk, NY, USA) was used for statistical analysis. Baseline classification data were expressed as percentages, and continuous data were expressed as medians or averages. The chi-square test was applied to categorical data, and Student's $t$-test or Wilcoxon $t$-test were applied to continuous data. Odds ratios (ORs) with 95\% confidence intervals (CIs) were assessed to determine the relationship 
TABLE 2 | Clinical outcomes and ischemia indicator.

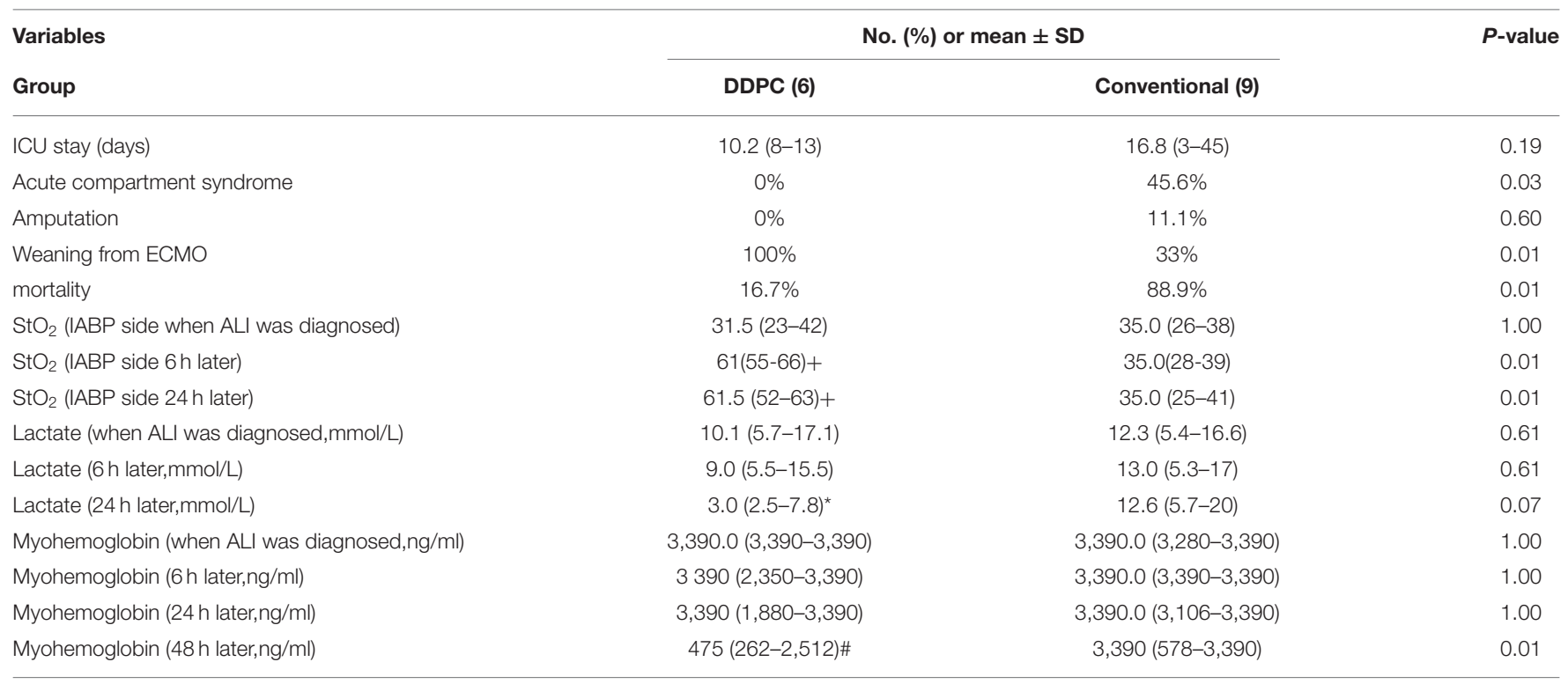

ALI, acute limb ischemia; $\mathrm{StO}_{2}$, tissue saturation.

*compare with the Lactate (when ALI was diagnosed).

+compare with the $\mathrm{StO}_{2}$ (when ALI was diagnosed).

\#compare with the myohemoglobin (when ALI was diagnosed).

between the changes in $\mathrm{StO}_{2}$, lactate and muscle injury markers. $P<0.05$ was considered a statistically significant difference.

\section{RESULTS}

The characteristics of the 15 patients with complications of limb ischemia on the IABP side are shown in Table 1. Normalized single tissue oxygen saturation values, lactate, and myohemoglobin when ALI was diagnosed are shown in Table 2. There was no significant difference between the 2 groups.

Overall, the in-hospital mortality for all patients was $60 \%$, with a mean SOFA score of $12.8 \pm 0.7$. The mortality rate was significantly lower in the DDPC group (16.7, vs. $88.9 \%, p=0.01)$. The ratio of successful ECMO weaning was significantly higher in the DDPC group than in the conventional group (100 vs. $33 \%, p=0.01$ ). Five patients in the conventional group developed osteofascial compartment syndrome and underwent incision and tensioning surgery ( 0 vs. $56 \% p=0.03)$. One patient was amputated in the conventional group ( 0 vs. $11.1 \% p=0.40$ ). Furthermore, no recurrence of lower limb ischemia was noted in any patients in the DDPC group.

$\mathrm{StO} 2$ was noticeably increased $6 \mathrm{~h}$ later in the DDPC group. The lactate level was significantly increased $24 \mathrm{~h}$ later in the DDPC group, and the myohemoglobin was significantly reduced $48 \mathrm{~h}$ later in the DDPC group. These parameters did not change significantly in the conventional treatment group (Table 2; Figure 2).

\section{DISCUSSION}

In this study, we described a method to manage severe ALI developing on the IABP side in inpatients with ECMO and IABP support. To our knowledge, this is the first study comparing the outcomes of DDPC with conventional treatment in these patients. Our results showed that DDPC is associated with lower mortality. It could also reduce the rate of acute compartment syndrome.

The combined use of IABP in ECMO patients may reverse protracted aortic valve closure and impaired left ventricular unloading $(11,12)$. It can also increase the cerebral blood flow (13). However, Chen et al. reported that the concomitant use of IABP with ECMO did not appear to be associated with a dramatic change in survival outcomes. It increased the incidence of lower limb ischemia (14). ALI on IABP side patients has a poor prognosis with high mortality and a high incidence of acute compartment syndrome, despite the removal of IABP (15).

Haldun et al. used polytetrafluoroethylene external femoro-femoral bypass grafting in patients assisted with ECMO and IABP (16). However, this approach is complex and demonstrates a higher incidence of infection and thrombogenesis. In this study, we report our experience with DDPC in 6 patients with adult PCS shock receiving VA-ECMO with limb ischemia on the IABP side. This procedure is simple, with no bleeding or infection complications in the groin. None of the patients developed osteofascial compartment syndrome, and none needed amputation. The $\mathrm{StO}_{2}$ was notably higher $6 \mathrm{~h}$ after DDPC. The markers of muscle injury peaked within $48 \mathrm{~h}$ with lower limb blood supply improvement. There were no significant differences in SOFA scores or Inotrope 


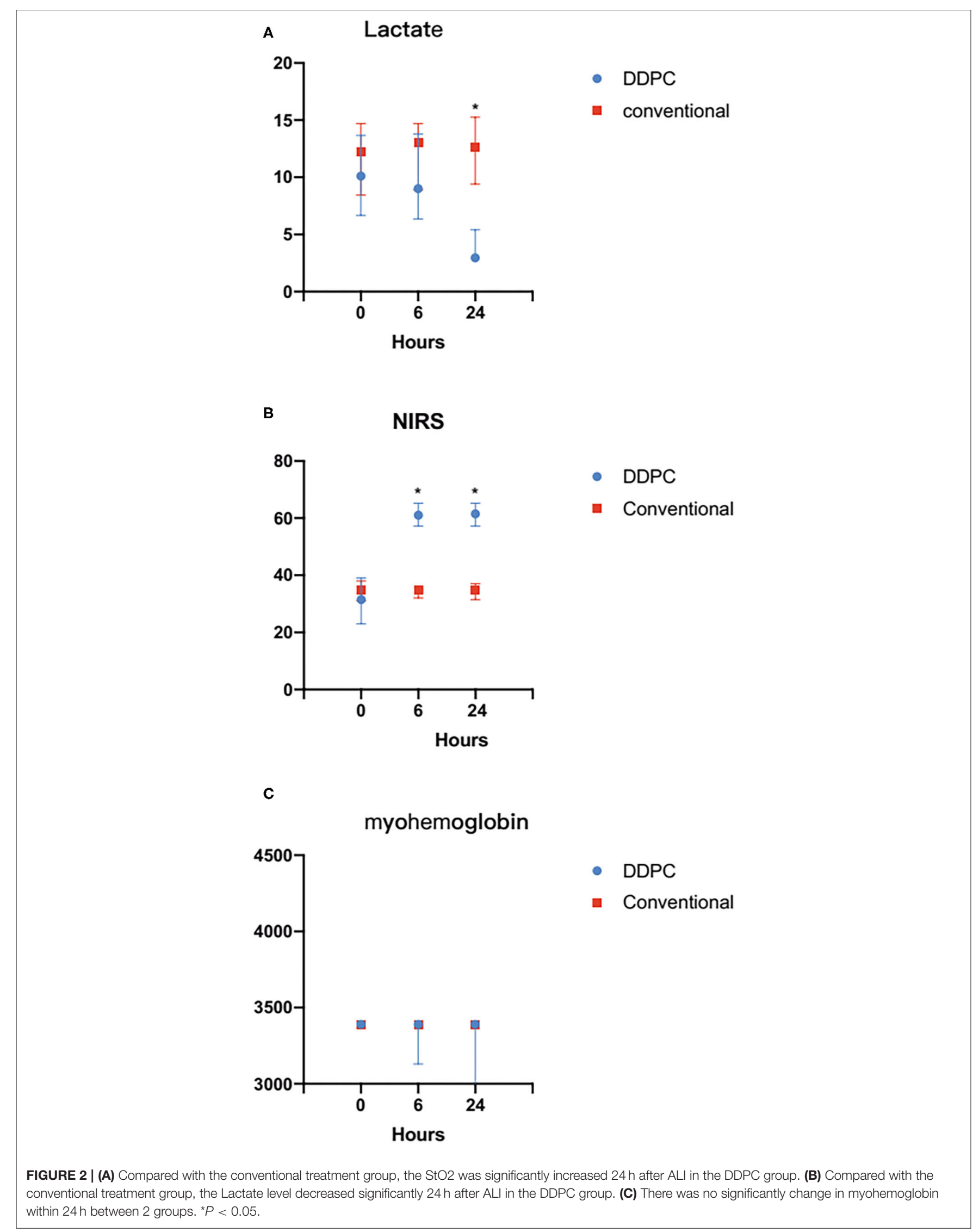


scores between the two groups, indicating that the severity of the disease was similar between the two groups before grouping. There was a significant difference in prognosis between the two groups, and the mortality was significantly reduced in the DDPC group. The StO2 of the DDPC group was significantly increased after DDPC placement, and myoglobin and lactate were also significantly decreased. The results suggested that this might be due to the improvement of lower limb ischemia.

DDPC could be removed safely while ECMO was weaning. Contraction of the peripheral vessels caused by the large doses of vasoactive agents and poor cardiac output may be the main cause of limb ischemia in the early ECMO stage. With the stability of the circulation system and reduced use of vasoactive agents, the blood supply of the lower limbs gradually recovers.

Our study suggests that DDPC is a simple, safe, and effective method. It may play a vital role in patients with complications of lower limb ischemia on the IABP side following ECMO combined with IABP.

The study has several limitations. First, it was a nonrandomized, retrospective, and observational study. The treatment strategies and monitoring methods that changed over time might have influenced the results of the study. Second, the number of patients included in this study was small, which may have prevented the detection of significant differences for other risk factors. Further multicenter studies are needed to corroborate the effectiveness of DDPC. Third, although pulse checks are still a routine diagnostic method for lower extremity ischemia in ECMO patients (17), advection perfusion of ECMO certainly has some influence on $\mathrm{ABI}$, and whether this index needs to be corrected in ECMO patients needs further study to confirm.

\section{REFERENCES}

1. Yau P, Xia Y, Shariff S, Jakobleff WA, Forest S, Lipsitz EC, et al. Factors associated with ipsilateral limb ischemia in patients undergoing femoral cannulation extracorporeal membrane oxygenation. Ann Vasc Surg. (2019) 54:60-5. doi: 10.1016/j.avsg.2018.08.073

2. Feng Y, Hou D, Wang J. Vascular complications in adult postcardiotomy cardiogenic shock patients receiving venoarterial extracorporeal membrane oxygenation. Ann Inten Care. (2018) 8:72-9. doi: 10.1186/s13613-018-0417-3

3. Gass A, Palaniswamy C, Aronow WS, Kolte D, Khera S, Ahmad H, et al. Peripheral venoarterial extracorporeal membrane oxygenation in combination with intra-aortic balloon counterpulsation in patients with cardiovascular compromise. Cardiology. (2014) 129:137-43. doi: $10.1159 / 000365138$

4. Nudcing S, Werdan K. IABP plus ECMO-Is one and one more than two? J Thorac Dis. (2017) 9:961-4. doi: 10.21037/jtd.2017.03.73

5. Lin LY, Liao CW, Wang CH, Chi NH, Yu HY, Chou NK, et al. Effects of additional intra-aortic balloon counter-pulsation therapy to cardiogenic shock patients supported by extra-corporeal membranous oxygenation. Sci Rep. (2016) 6:23838. doi: 10.1038/srep23838

6. Jang WJ, Cho YH, Park TK, Song YB, Choi JO, Hahn JY, et al. Fluoroscopyguided simultaneous distal perfusion as a preventive strategy of limb ischemia in patients undergoing extracorporeal membrane oxygenation. Ann Inten Care. (2018) 8:101-8. doi: 10.1186/s13613-018-0445-z

\section{CONCLUSION}

DDPC could be an effective method for lower limb ischemia on the IABP side in patients who received femoro-femoral VAECMO and IABP, and was associated with reduced mortality in these patients.

\section{DATA AVAILABILITY STATEMENT}

The original contributions presented in the study are included in the article/supplementary material, further inquiries can be directed to the corresponding author/s.

\section{DISCLOSURE}

All authors had freedom of investigation and full control of the design of the study, methods used, outcome parameters and results, analysis of data, and production of the written report.

\section{AUTHOR CONTRIBUTIONS}

MX is in charge of writing the articles. $\mathrm{XH}$ is in charge of providing idea. XT and LW are in charge of data analysis. HW, JW, and MJ are in charge of research design. DH is in charge of Chart production. All authors contributed to the article and approved the submitted version.

\section{FUNDING}

This project was funded by the National Key Research and Development Program of China (2016YFC1301001 to XH) and Beijing Municipal Science \& Technology Commission (No. Z161100000516017 to XH).

7. Lamb KM, DiMuzio PJ, Johnson A, Batista P, Moudgill N, McCullough $\mathrm{M}$, et al. Arterial protocol including prophylactic distal perfusion catheter decreases limb ischemia complications in patients undergoing extracorporeal membrane oxygenation. J Vasc Surg. (2017) 65:1074-9. doi: 10.1016/j.jvs.2016.10.059

8. Foley PJ, Morris RJ, Woo EY, Acker MA, Wang GJ, Fairman RM, et al. Limb ischemia during femoral cannulation for cardiopulmonary support. J Vasc Surg. (2010) 52:850-3. doi: 10.1016/j.jvs.2010.05.012

9. Norgren L, Hiatt WR, Dormandy JA, Nehler MR, Harris KA, Fowkes FG, et al. Inter-society consensus for the management of peripheral arterial disease (TASC II). J Vasc Surg. (2007) 43:61-7. doi: 10.1016/j.jvs.2006. 12.037

10. Li CL, Wang H, Jia M, Ma N, Meng X, Hou XT. The early dynamic behavior of lactate is linked to mortality in postcardiotomy patients with. Extracorporeal membrane oxygenation support: a retrospective observational study. J Thorac Cardiovasc Surg. (2015) 149:1445-50. doi: 10.1016/j.jtcvs.2014.11.052

11. Meani P, Delnoij T, Raffa GM, Morici N, Viola G, Sacco A, et al. Protracted aortic valve closure during peripheral veno-arterial extracorporeal life support: is intra-aortic balloon pump an effective solution? Perfusion. (2019) 34:35-41. doi: 10.1177/0267659118787426

12. Naito N, Nishimura T, Lizuka K, Morici N, Viola G, Sacco A, et al. Novel rotational speed modulation system used with venoarterial extracorporeal membrane oxygenation. Ann Thorac Surg. (2017) 104:1488-95. doi: 10.1016/j.athoracsur.2017.04.045 
13. Yang F, Jia ZS, Xing JL, Wang Z, Liu Y, Hao X, et al. Effects of intraaortic balloon pump on cerebral blood flow during peripheral venoarterial extracorporeal membrane oxygenation support. J Transl Med. (2014) 12:10614. doi: 10.1186/1479-5876-12-106

14. Chen R, Hachamovitch R, Makkar R, Ramzy D, Moriguchi JD, Arabia FA, et al. Lack of survival benefit found with use of intraaortic balloon pump in extracorporeal membrane oxygenation: a pooled experience of 1517 patients. J Invasive Cardiol. (2015) 27:453-8. doi: 10.1016/j.jacc.2014.07.096

15. Busch T, Sirbu H, Zenker D, Dalichau H. Vascular complications related to intraaortic balloon counterpulsation: an analysis of ten years experience. Thorac Cardiovasc Surg. (1997) 45:55-9. doi: 10.1055/s-2007-1013687

16. Haldun D, Maciej LD. External femorofemoral bypass to relieve acute leg ischemia during circulatory assist. Vascular. (2004) 12:198-201. doi: 10.1258/rsmvasc. 12.3.198

17. Chanan EL, Bingham N, Smith DE, Nunnally ME. Early detection, prevention, and management of acute limb ischemia in adults supported with venoarterial extracorporeal membrane oxygenation. J Cardiothorac Vasc Anesth. (2020) 34:3125-32. doi: 10.1053/j.jvca.2020.02.020
Conflict of Interest: The authors declare that the research was conducted in the absence of any commercial or financial relationships that could be construed as a potential conflict of interest.

Publisher's Note: All claims expressed in this article are solely those of the authors and do not necessarily represent those of their affiliated organizations, or those of the publisher, the editors and the reviewers. Any product that may be evaluated in this article, or claim that may be made by its manufacturer, is not guaranteed or endorsed by the publisher.

Copyright (c) 2021 Xin, Wang, Tian, Hou, Wang, Wang, Jia and Hou. This is an open-access article distributed under the terms of the Creative Commons Attribution License (CC BY). The use, distribution or reproduction in other forums is permitted, provided the original author(s) and the copyright owner(s) are credited and that the original publication in this journal is cited, in accordance with accepted academic practice. No use, distribution or reproduction is permitted which does not comply with these terms. 